\title{
Moonshot innovations: Wishful Thinking or Business-As-Usual?
}

\author{
Anne-Laure Mention \\ anne-laure.mention@rmit.edu.au | RMIT University, Australia \\ João José Pinto Ferreira \\ jjpf@fe.up.pt | INESC Technology and Science, Faculty of Engineering, University of Porto, Portugal \\ Marko Torkkeli \\ marko.torkkeli@lut.fi | Lappeenranta University of Technology, Finland
}

'Our mind-set will be to avoid the moonshot' said Boeing CEO James McNerney at a Wall Street analysts meeting in Seattle nearly 5 years ago (see Gates, 2014). The ambitious, exploratory and risky endeavour dubbed as moonshot project of the Boeing 787 Dreamliner had sunk billions of dollars in an industry where end-users demanded more comfort and convenience for less cost. According to McNerney, moonshots do not work in a price-sensitive environment. It is argued that they also tend to take the focus away from more immediate value capture opportunities as seen through Google's loss on its core Cloud Platform to Amazon Web Services (AWS). Google's parent company Alphabet which oversees Google X (a semi-secret moonshot project lab) more recently reported that it had incurred a US\$1.3billion in operating loss on moonshot projects with a sizeable increase in compensation of employees and executives working on these projects (Alphabet, 2018). Notably, none of the Google X lab spin-outs (e.g. Loon - a balloonbased internet project, Waymo - self-driving car project, Wing - drone delivery project) have been identified as commercially viable. Despite the uncertainties and failures, the focus on moonshot innovations continues to proliferate in academia (Kaur, Kaur and Singh, 2016; Strong and Lynch, 2018) and practice (Martinez, 2018). Yourden (1997) even wrote an interesting book on perseverance and tenacity to keep going even after failed projects. Proponents of moonshot thinking have claimed that it can help solve society's biggest challenges (e.g. cure cancer, see Kovarik, 2018) with some suggesting to encourage such thinking by paying failure bonuses (Figueroa, 2018). Yet others remain sceptical, positing that moonshot is 'awesome and pointless' (Haigh, 2019, p.4). A proverbial question, thus, emerges: are moonshot innovations simply wishful thinking or can they be part of business-as-usual? In part, the answer may be two-fold - 1) understanding the value of moonshot thinking, and 2) understanding moonshot challenges. 


\section{The value of moonshot thinking}

Perhaps the most talked about moonshot programs have come out of X, formerly Google X but now a separate identity under the parent company Alphabet. Its captain, Astro Teller, delivered a Ted talk on the "unexpected benefit of celebrating failure"1 in 2016. He identified two moonshot projects - automated vertical farming to tackle undernourishment and variable-buoyancy cargo ship to inexpensively transport cargo whilst reducing carbon footprint. Both these projects were killed as they failed to answer two inherent in all business-as-usual considerations - is it viable and feasible? The automated vertical farming could reduce water waste and save land resources but could not produce the much-needed staple crops like grains and rice. The variable buoyancy cargo ship in similar vein could reduce the need for runways and make an impact on carbon pollution but just to build one such ship for testing would cost nearly US $\$ 200$ million, money that Google X could not afford to burn at the time. Other X projects such as making affordable fuel from seawater and back-to-the-future style hoverboards with magnetic levitations have failed too. However, these setbacks did not stop Teller who promotes X as a moonshot factory that plans to spin-out innovations at the intersection of huge problems, breakthrough technologies and radical solutions (see Thompson, 2017). X boasts that it is not in the game of solving business-as-usual problems and that its purpose is to create the next Google by bringing together inter-disciplinary teams of inventors, engineers and makers.

To understand the value of moonshot thinking, one only needs to look at X's process and ideology. The most hopeful project from $\mathrm{X}$ is that of Waymo that aims to change land transportation through self-driving cars. In this talk Teller argued that building self-driving car came as a natural moonshot under the environment where nearly 1.2 million are dying on our roads each year. At one stage in 2018, Waymo was valued at US\$175billion by Morgan Stanley (Rapier, 2018) with other wall street firms claiming its worth to be nearing US $\$ 250$ billion (Ungarino, 2018). But of course, this all relies on the commercial viability of the self-driving cars and Waymo's other experiments on transport services (e.g. commercial freight, in-car services, etc.). In an interesting climax, soon after X retro-fitted Lexus into self-driving cars, they realised a major flaw in their moonshot thinking - once you make a driver less observant, s/he loses the attention to take back control in emergencies. The crisis meant Waymo was back to the drawing board, which eventually resulted in less ambitious (and arguably more viable) driver-engaged self-driving option. A more outrageous $\mathrm{X}$ project, which might be appropriately named, Loon promised a network of balloons floating in the air to provide internet to remote and rural places. However, after being sued for patent infringement, misappropriation of trade secrets, breach of contract and losing critical technology rights to rival firm Space Data, the Loon balloon might just have popped (pun intended) (see Harris, 2017). As if he had a crystal ball, Bill Gates in an interview with Bloomberg five years earlier had said such projects do not help solve core societal problems. He added, "when you are dying of malaria, I suppose you'll look up and see that balloon, and I'm not sure how it'll help you. When a kid gets diarrhea, no, there's no website that relieves that. Google started out saying they were going to do a broad set of things and they shut it all down. Now they are just doing their core thing" (Stone, 2013). Loon and Waymo

$1 \quad$ See the full transcript of Astro Teller's Ted 2016 talk here: https://www.ted.com/talks/astro_teller_ the_unexpected_benefit_of_celebrating_failure/transcript 
raise an important question, does moonshot thinking really help solve problems or has $\mathrm{X}$ has completely misunderstood what moonshot is? The quest to answer this question needs a quick lesson in history - the real moonshot story.

J.F. Kennedy stood at Rice University in 1961 and announced that he had a dream, a dream to put man on the moon and safely bring him back. The intent, planning, mental effort and execution of what followed eight years later as the Apollo 11 mission came to be known as the moonshot project. Apollo 11 heralded three men into space, landed two on the moon who took some pictures and picked up rocks and, eventually returned all of them back to earth in a compact capsule. Apollo 11 marked a giant leap in technological projects is noted as a triumph for a new era in which coordinated management systems are designed and integrated with sophisticated engineering to create successful moonshots. It showcased America's political will, technological prowess and economic capacity in the age of space race. The real moonshot was neither economically viable, nor practically feasible at the time, a far cry from X's current take on its moonshot innovations. Apollo program united the nation, forged new collaborations and pushed the existing technologies to new levels of performance and reliability - the unintended benefit of moonshot thinking. Indeed, several new technologies emerged as spin-offs from lessons learned from Apollo - Velcro and Teflon being the prominent ones amongst others that brought us freeze-fried raspberries, scratch-resistant eyewear and featherlight foil blankets. Besides Apollo itself created numerous new jobs and redefined computer engineering techniques that resulted in new microchips and information management systems (Haigh, 2009). Apollo shaped our future in radical ways - from catapulting space science to environmental movements to the utopia of the emerging tech culture (Turner, 2006). A key lesson from Apollo program is that moonshot innovations do not start with clever answers, rather they start with the painstaking task of finding the right questions. On this logic, perhaps moonshot and innovations do not belong together. Loon and Waymo have not failed if the iterations in their experimental stages resulted in deeper discussions on how humans interact with technologies and if the subsequent focus continues to be afforded on the development cycles. However, Teller's approach of failing fast and cheap seems too far from the moonshot that Apollo was. As Haigh (2017) argues, 'Letting Silicon Valley steal the term "moonshot" for projects with quite different management styles, success criteria, scales, and styles of innovation hurts our collective ability to understand just what NASA achieved 50 years ago and why nothing remotely comparable is actually under way today at Google, or anywhere else' (p. 2).

\section{Moonshot challenges}

The question is how we catalyse moonshot thinking while still focusing on viability and feasibility? While innovations tend to stretch the boundaries of strategy and human cognition (see Sund, Galavan and Brusoni, 2018), moonshots galvanise communities towards tackling a huge societal challenge and shape desired future in the process. Inherently, all moonshot innovations have common traits - they are risky, sound outrageous for the time, resource consuming and call for some of the best minds to collaborate for months and years. They are more than where a company executive wants to take the firm, almost certainly more than the desire to create new products. Understandably so, all moonshots are radical innovations but not all radical 
innovations can claim to be moonshoots - the defining keywords being 'huge societal challenge' and 'shape desired future'.

Cancer is a huge societal challenge and so is clean energy, internet access not as much. Companies such as Grail that is working to detect and cure cancer before its symptoms become evident, are the ones that can claim moonshot innovations. Grail is testing new ways to conduct liquid biopsy and search for cancerous mutations that can be detected with any available technology. Likewise, apart from the obvious, a huge challenge for today's urbanised society is to create construction material with low CO2 emissions. Companies such as bioMason have developed bricks with materials that do not require heat to produce, using microorganisms to grow biocement - an outrageous proposition for the prevailing traditional masonary techniques. There are several such examples, from Tesla's home batteries to Sungevity's remote solar design tool to the simpler food alternatives to animal-based products ${ }^{2}$.

Notable observations amongst these examples are that in striving for moonshots, the firms did not abandon the attention towards incremental (or the so called 10\%) improvements. Businessas-usual remains focused on the core activities at these firms - developing quick fail prototypes, sourcing and combining emerging technologies and looking for those quick-wins and revenue growth bets. This attention is important as by its very definition, moonshot calls for a long-term futuristic vision, looking beyond the present affordances and imagining a desired world that may never eventuate. For managers and captains of moonshot, the proverbial saying that "shoot for the moon and fall amongst the stars"may present just the right amount of pragmatism to moonshot thinking. Yet, so as far as firms continue to rely on return on investments, a prudent strategy will always be to maintain the business-as-usual through a portfolio of new ventures. The probability of landing amongst the stars is certainly higher than landing on the moon. Coming back to the opening of this editorial, McNerney said he would rather have Boeing innovate like Apple than do moonshots every 25 years. He was of course referring to period between 20062010, the peak of iPhone development when Apple spend just US $\$ 4.6$ billion against Microsoft's US $\$ 31$ billion to shape the future of telecommunications. Moonshot innovations, at least in their current state, are simply wishful thinking and far from business-as-usual. This is disturbing since the value of moonshot is far reaching (once again pun intended) but in the age of corporate venturing, uncertainties and complex decision contexts, the pressure of viability and feasibility is continual - for, if you have promised to shoot for the moon, you better hit it.

Innovatively yours,

Anne-Laure Mention, João José Pinto Ferreira, Marko Torkkeli

The Editors

\section{References}

Alphabet, 2018. Fourth quarter fiscal year 2018 results. Available at: https://abc.xyz/investor/static/pdf/2018Q4_alphabet_earnings_release.pdf?cache=adc3b38

$2 \quad$ See the JUST stories at https://www.ju.st/en-us/stories 
Figueroa, J., 2018. Failure Bonuses: Incentives for a Moonshot Factory. Available at: https://scholar.princeton.edu/sites/default/files/josuefigueroa/files/failurebonuses.pdf

Gates, D., 2014. McNerney: No more 'moonshots' as Boeing develops new jets. Seattle Times, Business/Technology, 21 May. Available at:

http://old. seattletimes.com/html/businesstechnology/2023668895_boeinginvestorconf xml. html

Haigh, T., 2009. How data got its base: Information storage software in the 1950s and 60s. IEEE Annals of the History of Computing, 31 (4), pp. 6-25.

Haigh, T., 2019. Hey Google, what's a moonshot?: how Silicon Valley mocks Apollo. Communications of the ACM, 62(1), pp.24-30.

Harris, M., 2017. The lawsuit that could pop Alphabet's project look balloons. Wired, 7 May. Available at:

https://www.wired.com/story/the-lawsuit-that-could-pop-alphabets-project-loon-balloons/

Kaur, K., Kaur, R. and Singh, J.S., 2016. Use of Moonshot Thinking in Space Operational Robotics (SOR)-A Theoretical Analysis. International Journal of Computer Applications, $155(2)$.

Kovarik, J.E., 2018. Cancer Moonshot: Patents for Patients. Trends in cancer, 4(8), pp.515516.

Martínez, M.E. and Paskett, E.D., 2018. Using the Cancer Moonshot to Conquer Cancer Disparities: A Model for Action. JAMA oncology, 4(5), pp.624-625.

Rapier, G., 2018. Waymo is worth $\$ 100$ billion more than previous estimates, Morgan Stanley says. Markets Insider, 7 August. Available at:

https://markets.businessinsider.com/news/stocks/google-stock-price-waymo-worth-100-billion-m utm_source=markets\&utm_medium=ingest

Strong, A.C. and Lynch, C., 2018. Learning from a moonshot: What's next for college summer reading?. New England Journal of Higher Education. Available at:

https://nebhe.org/journal/learning-from-a-moonshot-what-next-for-college-summer-reading/

Stone, B., 2013. Bill Gates on his foundation's health and education campaigns. Bloomberg Business, 9 August. Available at:

https://www.bloomberg.com/news/articles/2013-08-08/bill-gates-on-his-foundations-health-and$\mathrm{p} 1$

Sund, K.J., Galavan, R.J., and Brusoni, S., 2018. Cognition and innovation. Emerald Publishing, United Kingdom.

Thompson, D., 2017. Google X and the science of radical creativity. The Atlantic, November. Available at:

https://www . theatlantic.com/magazine/archive/2017/11/x-google-moonshot-factory/540648/

Turner, F., 2006. From Counterculture to Cyberculture: Stewart Brand, the Whole Earth Network, and the Rise of Digital Utopianism, University of Chicago Press, Chicago. 
Ungarino, R., 2018. Waymo could be a $\$ 250$ billion win for Alphabet. Markets Insider, 19 December. Available at:

https://markets.businessinsider.com/news/stocks/alphabet-stock-waymo-could-be-a-250-billion-

Yourdon, E., 1997. Death march: The complete sofware developer's guide to surviving "Mission Impossible" projects, Prentice Hall.

Cite paper as: Mention, A., Pinto Ferreira, J.J., Torkkeli, M., (2019). Moonshot innovations: Wishful Thinking or Business-As-Usual?, Journal of Innovation Management, www.open-jim.org, 7(1), 1-6. HANDLE: https://hdl.handle.net/10216/119830; DOI: https://doi.org/10.24840/2183-0606_007.001_0001 